\title{
The molecular gas around the luminous blue variable star G24.73+0.69
}

\author{
A. Petriella ${ }^{1,2}$, S. A. Paron ${ }^{1,3}$, and E. B. Giacani ${ }^{1,3}$ \\ 1 Instituto de Astronomía y Física del Espacio (CONICET-UBA), CC 67, Suc. 28, 1428 Buenos Aires, Argentina \\ e-mail: apetriella@iafe.uba.ar \\ 2 CBC - Universidad de Buenos Aires, Argentina \\ 3 FADU - Universidad de Buenos Aires, Argentina
}

Received 3 November 2011 / Accepted 22 November 2011

\section{ABSTRACT}

\begin{abstract}
Aims. We study the molecular environment of the luminous blue variable star G24.73+0.69 to investigate the origin of the two infrared shells around this massive star and determine its effects on the surrounding interstellar medium.

Methods. We analyze the distribution of the molecular gas using the ${ }^{13} \mathrm{CO} J=1-0$ emission extracted from the Galactic Ring Survey. We use near- and mid-infrared data from 2MASS and GLIMPSE to identify the young stellar objects in the field.

Results. We discover the molecular counterpart to the outer infrared shell around G24.73+0.69. The CO shell was probably blown by the stellar wind of the star mainly during its main sequence phase. We also find molecular gas that corresponds to the inner infrared shell, although its origin remains uncertain. We identify seven young stellar objects within the molecular material, whose birth might have been triggered by the stellar wind of the luminous blue variable star. We suggest that both G24.73+0.69 and the progenitor of the nearby supernova remnant G24.7+0.6 were formed from the same natal cloud and represent the most evolved members of a so far undetected cluster of massive stars.
\end{abstract}

Key words. stars: individual: G24.73+0.69 - stars: winds, outflows - ISM: clouds - stars: formation

\section{Introduction}

Luminous blue variable (LBV) stars are very massive objects that evolve from O-type main sequence (MS) stars burning hydrogen in their core to become Wolf-Rayet (WR) helium core burning stars (Maeder 1989; Humphreys et al. 1989). This transitional stage is characterized by a high mass-loss rate (typically between $10^{-5}$ and $10^{-4} M_{\odot} /$ yr) sometimes accompanied by so-called giant eruptions, and significant photometric variability on timescales from months to years (Humphreys \& Davidson 1994). The LBV phase is short-lived and as a consequence only 12 cases and 23 candidates have been reported in our Galaxy so far (Clark et al. 2005). As a result of the mass loss, most of the LBV stars (either confirmed or candidates) are surrounded by a nebula that expands with characteristic velocities of between $30 \mathrm{~km} \mathrm{~s}^{-1}$ and $200 \mathrm{~km} \mathrm{~s}^{-1}$ (see Clark et al. 2005). These nebulae have dusty and gaseous components (of a few solar masses) and a large variety of morphologies, from circular to bipolar (Nota et al. 1995; Weis 2001).

Since nebulae around LBV stars are strong emitters in the infrared (IR) regime, most of them have been observed in this wavelength range. At present, there are only a few cases in which the chemical evolution and the presence of circumstellar molecular gas related to these nebulae have been determined by molecular studies, the most representative sources being AG Car (Nota et al. 2002) and G79.29+0.46 (Rizzo et al. 2008; Jiménez-Esteban et al. 2010). Molecular material was also discovered around the yellow hypergiant (YHG) stars IRC +10420 and AFGL2343* (Castro-Carrizo et al. 2007). Theoretical models of stellar evolution and observations of post-MS massive stars (Meynet \& Maeder 2000; Humphreys \& Davidson 1994) suggest that low luminous LBVs may pass through a cooler red supergiant (RSG)/yellow hypergiant phase prior to the LBV period. The RSG/YHG phase is also characterized by mass-loss activity (for a review, see de Jager 1998) that can lead to the presence of molecular gas around these massive stars. By analyzing the molecular material, we can determine the mass-loss history of the massive star and its effects on the surrounding interstellar medium (ISM).

\section{Presentation of $\mathrm{G} 24.73+0.69$}

The ring nebula around the LBV star G24.73+0.69 (hereafter G24) was discovered by Clark et al. (2003) in the mid-IR. Figure 1 displays the emission in the Spitzer-IRAC $8 \mu \mathrm{m}$ band toward G24, which clearly shows the nebula around the central massive LBV star. It has a roughly circular morphology with a slight elongation in a direction parallel to the Galactic plane. Its shape can be approximated by an ellipse of $40^{\prime \prime} \times 34^{\prime \prime}$. Taking an expansion velocity of $200 \mathrm{~km} \mathrm{~s}^{-1}$, Clark et al. (2003) estimated a mass-loss rate of $\sim 9.5 \times 10^{-5} M_{\odot} / \mathrm{yr}$ for the LBV star and an age of $\sim 4800 \mathrm{yr}$ for the nebula. They quoted an upper limit of $5.2 \mathrm{kpc}$ for the distance based on the consistency between the modeled and the observed spectral energy distribution of the star. In addition, there is a fragmentary outer shell (OS) of a bipolar morphology with lobes (labeled 1 and 2 in Fig. 1) projecting into opposite directions. This structure forms an incomplete shell of $4.6^{\prime} \times 2.3^{\prime}$ oriented $\sim 60^{\circ}$ with respect to the Galactic plane, whose geometric center does not match the position of the LBV star. Figure 1 also shows the position of the IR dark cloud (IRDC) $024.789+0.633$, which is adjacent to the lobe 2. All IRDCs are considered as the precursors of protostars, being a signature of active star formation (Rathborne et al. 2006, 2007). 


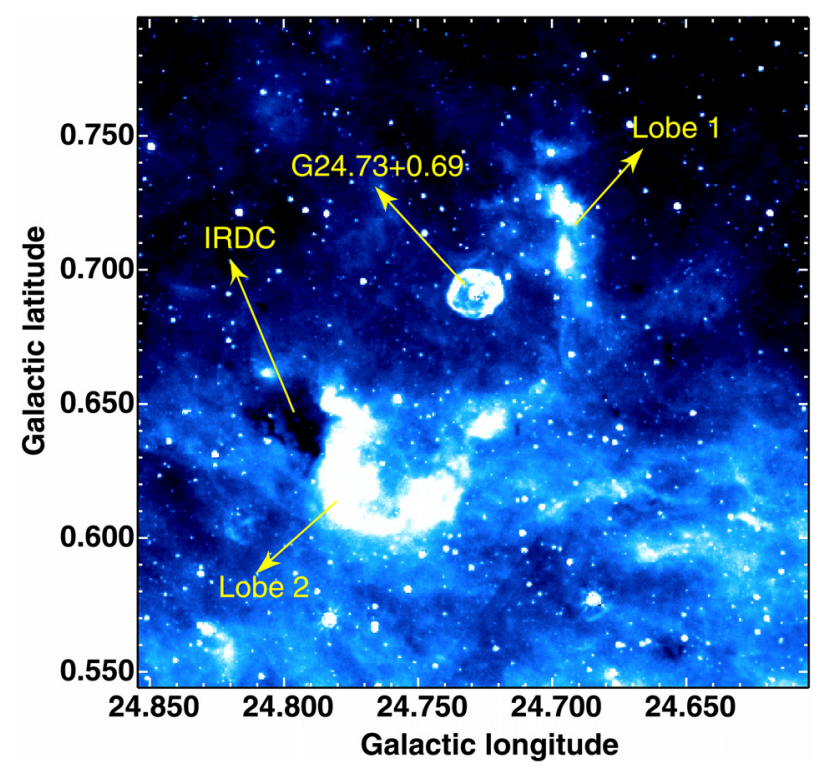

Fig. 1. Emission in the Spitzer-IRAC $8 \mu \mathrm{m}$ band toward the LBV star G24.73+0.69. We indicate the positions of the LBV star and its nebula, the lobes of the bipolar outer shell, and the IRDC 024.789+0.633.

To explain the origin of the IR shells, Clark et al. (2003) argued that the inner one (i.e. the ring nebula) originates in material ejected from the central star during the LBV phase. However, based on the size and age of the bipolar OS, they suggested that it formed from the interaction between the stellar wind and the ISM and/or the natal molecular cloud (MC) in a period prior to the current phase.

In this work, we study the molecular gas toward G24.73+0.69 and its environment with the aim of unveiling the origin of these IR shells.

\section{Results and discussion}

\subsection{The distribution of the molecular gas}

We study the molecular gas around G24 using data from the Galactic Ring Survey (GRS, Jackson et al. 2006). The GRS was performed by the Boston University and the Five College Radio Astronomy Observatory (FCRAO). The survey maps the Galactic ring in the ${ }^{13} \mathrm{CO} J=1-0$ line with an angular and spectral resolutions of $46^{\prime \prime}$ and $0.2 \mathrm{~km} \mathrm{~s}^{-1}$, respectively. The observations were performed in both position-switching and on-the-fly mapping modes, achieving an angular sampling of $22^{\prime \prime}$.

From the inspection of the ${ }^{13} \mathrm{CO}$ data cube in the whole velocity range, we found morphological signatures of a possible association between G24 and the surrounding molecular material in the velocity range between +39 and $+44 \mathrm{~km} \mathrm{~s}^{-1}$ (all velocities here being referred to the local standard of rest). In Fig. 2, we show the integrated velocity channel maps of the ${ }^{13} \mathrm{CO} J=1-$ 0 emission every $0.7 \mathrm{~km} \mathrm{~s}^{-1}$ with the mid-IR emission from the Spitzer-IRAC $8 \mu \mathrm{m}$ band. From this figure, we can discern several condensations of molecular gas forming a molecular shell around G24 (see mainly panels at +40.9 and $+41.5 \mathrm{~km} \mathrm{~s}^{-1}$ ). This shell delineates the exterior border of the bipolar OS. This distribution indicates that there is a connection between the molecular gas and the bipolar OS. If this were the case, both of them would be at the same distance. Using the rotational model of Fich et al. (1989) and adopting a velocity of $+42 \mathrm{~km} \mathrm{~s}^{-1}$ as a systemic velocity for the molecular shell, we obtained kinematic distances of either $3.5 \mathrm{kpc}$ or $12 \mathrm{kpc}$. Lacking any additional discriminator between the two kinematic distances and taking into account that a distance of $5.2 \mathrm{kpc}$ is quoted as an upper limit for G24 (Clark et al. 2003), we adopted a distance of $3.5 \mathrm{kpc}$ as the most plausible value for the LBV star and the molecular shell. This new distance led to a substantial reduction in the calculated intrinsic luminosity of G24. Clark et al. (2003) quoted $\log \left(L_{\star} / L_{\odot}\right)=5.6$ for a distance of $5.2 \mathrm{kpc}$. Taking a distance of $3.5 \mathrm{kpc}$, the luminosity drops to $\log \left(L_{\star} / L_{\odot}\right) \sim 5.25$, which would make it one of the faintest LBVs yet identified (see for comparison the HR diagram of Clark et al. 2009), thus a possible post RSG/YHG object.

We were able to derive a number of parameters characterizing the $\mathrm{CO}$. By assuming local thermodynamic equilibrium (LTE), we estimated the $\mathrm{H}_{2}$ mass of the molecular shell using the equations

$N\left({ }^{13} \mathrm{CO}\right)=2.42 \times 10^{14} \frac{T_{\mathrm{ex}}+0.88}{1-\mathrm{e}^{-5.29 / T_{\mathrm{ex}}}} \int \tau_{13} \mathrm{~d} v$,

where $T_{\text {ex }}$ is the excitation temperature of the ${ }^{13} \mathrm{CO}$ transition and $\tau_{13}$ is the optical depth of the line. Assuming that the ${ }^{13} \mathrm{CO}$ $J=1-0$ line is optically thin, we can use the approximation

$\int \tau_{13} \mathrm{~d} v \sim \frac{1}{J\left(T_{\mathrm{ex}}\right)-J\left(T_{\mathrm{b}}\right)} \int T_{\mathrm{B}} \mathrm{d} v$,

where

$J(T)=\frac{5.29}{\mathrm{e}^{5.29 / T}-1}$,

$T_{\mathrm{b}}=2.7 \mathrm{~K}$ is the background temperature, and $T_{\mathrm{B}}$ is the brightness temperature of the line. We assumed that $T_{\mathrm{ex}}=10 \mathrm{~K}$ and used the relation $N\left(\mathrm{H}_{2}\right) / N\left({ }^{13} \mathrm{CO}\right) \sim 5 \times 10^{5}$ (Simon et al. 2001) to estimate the column density of molecular hydrogen $N\left(\mathrm{H}_{2}\right)$. The mass of the molecular clumps was calculated from

$M=\mu m_{\mathrm{H}} \sum\left[D^{2} \Omega N\left(\mathrm{H}_{2}\right)\right]$,

where $\Omega$ is the solid angle subtended by the ${ }^{13} \mathrm{CO} J=1-0$ beam size, $m_{\mathrm{H}}$ is the hydrogen mass, $\mu$ is the mean molecular weight assumed to be 2.8 by taking into account a relative helium abundance of $25 \%$, and $D \sim 3.5 \mathrm{kpc}$ is the distance. Our summation was performed over the area of each molecular clump. We summed the mass of the clumps around G24 and found that the molecular shell has a total mass of $\sim 1000 M_{\odot}$. The original ambient density $n_{0}$, an important parameter to study the dynamics of the shell, could be estimated by assuming that the calculated total mass was originally distributed in the volume limited by the bipolar OS, which has a mean radius of $\sim 3.5^{\prime}$. At a distance of $3.5 \mathrm{kpc}$, this corresponds to a mean radius $r_{0} \sim 3.5 \mathrm{pc}$. Using this value, we found that $n_{0} \sim 230 \mathrm{~cm}^{-3}$.

\subsection{The origin of the molecular shell}

There is compelling evidence of shells of interstellar material produced by the effect of the strong stellar winds of massive stars on the ISM. These so-called stellar wind bubbles have been observed in neutral (Cappa et al. 2005; Cichowolski et al. 2008; Giacani et al. 2011) and molecular (Cappa et al. 2009, 2010) gas. We explored the hypothesis that the action of the stellar wind of the LBV star and/or its progenitor O-type star has blown a cavity of interstellar material piling up the molecular gas in the border and forming the molecular shell discovered in the present work. 

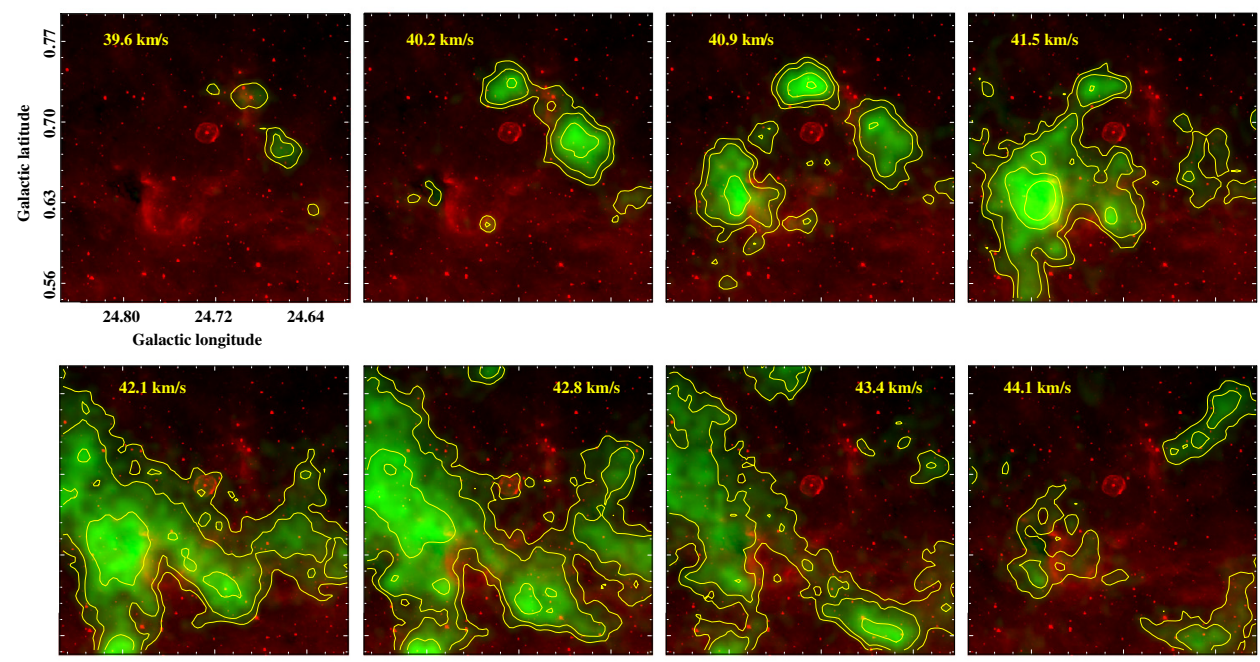

Fig. 2. Two-color image of the field around the LBV star. In red: Spitzer-IRAC $8 \mu \mathrm{m}$ band. In green: the integrated ${ }^{13} \mathrm{CO} J=1-0$ emission every $0.7 \mathrm{~km} \mathrm{~s}^{-1}$. The $\mathrm{CO}$ contour levels are $0.5,0.9,1.8$, and $2.7 \mathrm{~K} \mathrm{~km} \mathrm{~s}^{-1}$.

To test this scenario, we compared the mechanical energy $E_{\omega}$ released by the star into the ISM during the lifetime of the molecular shell, with the kinetic energy $E_{\mathrm{k}}$ of the sweptup material. We first estimated the dynamical age $\left(t_{\text {dyn }}\right)$ of the shell using the equation of Whitworth et al. (1994) for a stellar wind bubble: $t_{\mathrm{dyn}} \sim 0.02 r_{0}^{5 / 3} L_{37}^{-1 / 3} n_{3}^{1 / 3} \mathrm{Myr}$, where $r_{0}$ is the radius of the shell in pc, $L_{37}$ is the mechanical luminosity $L_{\omega}$ of the stellar wind in units of $10^{37} \mathrm{erg} \mathrm{s}^{-1}$, and $n_{3}$ is the original ambient density in units of $10^{3} \mathrm{~cm}^{-3}$. We calculated $L_{\omega}=1 / 2 \dot{M} v_{\omega}^{2}$ by taking the typical parameters of an O-type star in Prinja et al. (1990) and Mokiem et al. (2007), namely a mass-loss rate $\dot{M}=2 \times 10^{-6} M_{\odot} /$ yr and a stellar wind velocity $v_{\omega}=2000 \mathrm{~km} \mathrm{~s}^{-1}$. Thus, we found that $L_{\omega} \sim 2.5 \times 10^{36} \mathrm{erg} \mathrm{s}^{-1}$. Taking the mean radius of the shell $\sim 3.5 \mathrm{pc}$ and the original ambient density $\sim 230 \mathrm{~cm}^{-3}$, we evaluated the $t_{\text {dyn }}$ to be $\sim 0.2 \mathrm{Myr}$. From this result, we can see that the $\mathrm{CO}$ shell is more than an order of magnitude older than the age of the LBV phase, which typically has a duration of $\lesssim 10^{4} \mathrm{yr}$. This indicates that the detected CO shell may be the material swept-up by the stellar wind of the central massive star during its MS phase.

The kinetic energy can be estimated as $E_{\mathrm{k}}=1 / 2 M v_{\mathrm{e}}^{2}$, where $M$ is the mass of the shell and $v_{\mathrm{e}}$ its expansion velocity. We calculated the expansion velocity to be $v_{\mathrm{e}} \sim$ $6.2 L_{37}^{1 / 5} n_{3}^{-1 / 5} t_{\text {dyn }}^{-2 / 5} \mathrm{~km} \mathrm{~s}^{-1}$ (Whitworth et al. 1994), where $t_{\text {dyn }}=$ 0.2 Myr. We obtained $v_{\mathrm{e}} \sim 13 \mathrm{~km} \mathrm{~s}^{-1}$. Using $M=1000 M_{\odot}$, we inferred that $E_{\mathrm{k}} \sim 1.7 \times 10^{48} \mathrm{erg}$. For the mechanical energy, we assume that $E_{\omega}=L_{\omega} t_{\text {dyn }} \sim 1.6 \times 10^{49} \mathrm{erg}$. We calculated the efficiency of the energy conversion of the wind $\epsilon=\frac{E_{\mathrm{k}}}{E_{\mathrm{e}}}$ and obtained a value of $\sim 0.11$. This agrees with the expected value for an energy-conserving stellar wind bubble (Mellema \& Lundqvist 2002), hence we conclude that the central star has blown a stellar wind strong enough to create the molecular shell. We also evaluated the contribution of the star during the LBV phase to the energy outflow. Taking $\dot{M}=9.5 \times 10^{-5} M_{\odot} / \mathrm{yr}$ and $v_{\omega}=$ $200 \mathrm{~km} \mathrm{~s}^{-1}$ from Clark et al. (2003) and $\sim 10^{4} \mathrm{yr}$ as a typical duration for the LBV phase, we obtained $E_{\omega}^{\mathrm{LBV}} \sim 3.8 \times 10^{47} \mathrm{erg}$, which represents less than $3 \%$ of the energy released by the star during the MS phase.

These calculations link the formation of the molecular shell to the stellar wind of the central star during the MS phase, in good agreement with the origin of the bipolar OS suggested by Clark et al. (2003). In this scenario, the elongated morphology of the bipolar OS (and also of the molecular shell) may be a consequence of the asymmetric stellar wind from the central star. Evidence of non-spherical winds has been found in other massive stars. For example, Meaburn et al. (2004) discovered a giant lobe projecting from the ring nebula around the LBV star P Cygni. According to the authors, the lobe formed from the massejection activity prior to the current eruptions that produced the nebula. They suggested that the peculiar morphology of this giant lobe is due to the interaction between the ejected material and an asymmetric cavity in the ISM blown by the toroidal stellar wind of the central star during the MS phase.

Finally, we note the presence of molecular gas superimposed on the ring nebula (see Fig. 2, panels at +42.1 and $+42.8 \mathrm{~km} \mathrm{~s}^{-1}$ ). This material may i) originate in a mass-ejection event from the central LBV star, as observed in the ring nebula around G79.29+0.46 (Jiménez-Esteban et al. 2010); or ii) be part of the molecular shell. Using the equations of Sect. 3.1, we estimated a mass of $\sim 20 M_{\odot}$ and a density of $\sim 120 \mathrm{~cm}^{-3}$. The obtained mass is higher than the mass of the nebula around G24 estimated by Clark et al. (2003) $\left(\sim 0.45 M_{\odot}\right)$ and of the nebulae around other LBVs (see Smith \& Owocki 2006 and Clark et al. 2009). Thus, we propose that the molecular material located right upon the ring nebula belongs mainly to the molecular shell. However, we cannot rule out the presence of some molecular gas ejected by the LBV star. We plan to observe a field around G24 with higher density tracers and higher resolution to confirm the presence of this material.

\subsection{Star formation activity around G24.73+0.69}

The environment of the LBV star G24 might be one that promotes triggered star formation. As we have shown in the previous section, the massive star has been interacting with the neighboring molecular gas by means of its strong stellar wind. To search for primary tracers of star formation, we identified the young stellar object (YSO) candidates using the GLIMPSE Point Source Catalog (GPSC) in the Spitzer-IRAC bands. We constructed a [5.8]-[8.0] versus [3.6]-[4.5] color-color (CC) diagram for the sources around G24. In the CC diagram, we used the photometric criteria of Allen et al. (2004) to classify the sources according to their evolutionary stage: class I are 
Table 1. 2MASS and Spitzer-IRAC magnitudes of class I YSO candidates.

\begin{tabular}{lccccccccc}
\hline \hline Source & Source (GPSC) & 2MASS Qual. & $J$ & $H$ & $K_{\mathrm{S}}$ & $3.6 \mu \mathrm{m}$ & $4.5 \mu \mathrm{m}$ & $5.8 \mu \mathrm{m}$ & $8.0 \mu \mathrm{m}$ \\
\hline 1 & G024.7956+00.6558 & N/D & & & & 13.047 & 12.173 & 11.715 & 11.321 \\
2 & G024.8051+00.6456 & UUA & 18.131 & 17.059 & 13.707 & 10.548 & 9.589 & 8.791 & 8.059 \\
3 & G024.7901+00.6410 & N/D & & & & 12.874 & 11.096 & 9.983 & 9.639 \\
4 & G024.7907+00.6379 & N/D & & & & 13.439 & 12.518 & 11.638 & 11.059 \\
5 & G024.7545+00.6771 & AAA & 14.818 & 13.227 & 12.158 & 10.967 & 10.497 & 10.194 & 9.859 \\
6 & G024.7532+00.6400 & UUA & 14.643 & 12.784 & 12.312 & 11.792 & 11.463 & 11.722 & 11.386 \\
7 & G024.7735+00.6068 & AAA & 13.898 & 13.172 & 12.803 & 11.907 & 11.485 & 10.083 & 8.577 \\
\hline
\end{tabular}

Notes. 2MASS Qual.: A is the best photometric quality, with a $S N R \geq 10$, and U means that the magnitude value is an upper limit. N/D indicates no detection of the source in the 2MASS survey.

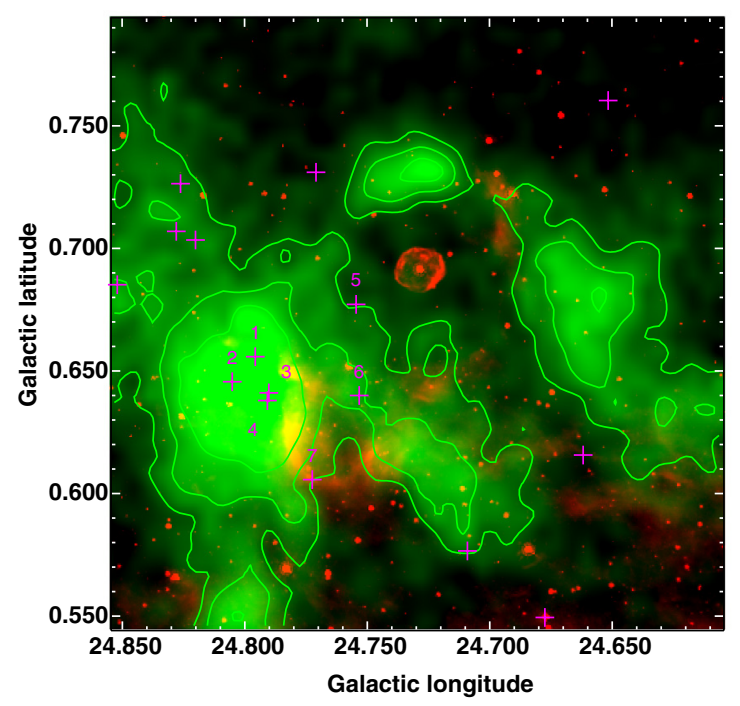

Fig. 3. Two-color image toward G24.73+0.69. In green with contours: emission of the ${ }^{13} \mathrm{CO}$ integrated between +39 and $+44 \mathrm{~km} \mathrm{~s}^{-1}$ (contour levels are 2.7, 4.4, and $6.2 \mathrm{~K} \mathrm{~km} \mathrm{~s}^{-1}$ ). In red: $8 \mu \mathrm{m}$ band. The magenta crosses are the class I YSO candidates selected according to the criterion discussed in the text.

protostars with prominent circumstellar envelopes, class II are disk-dominated objects, and class III are the most evolved YSOs whose emission comes mainly from the central star.

Figure 3 displays the distribution of the class I YSO candidates around G24. We can discern seven of these YSO candidates superimposed on the molecular gas swept-up by the massive star. Four of them (sources 1, 2, 3, and 4) spatially coincide with the maximum of molecular emission. We note that this region coincides with the IRDC $024.789+0.633$ (indicative of active star-forming activity) and lies next to the lobe 2 of the bipolar OS.

We fit the spectral energy distribution (SED) of the class I YSO candidates using the fluxes in the 2MASS $J, H$, and $K \mathrm{~s}$ bands and in the four Spitzer-IRAC bands. We used the tool developed by Robitaille et al. (2007) and available online ${ }^{1}$. We assumed the distance to be between 3 and $4 \mathrm{kpc}$. In Table 1, we report the class I YSO candidates magnitudes in the 2MASS and Spitzer-IRAC bands. Robitaille et al. (2006) defined three evolutionary stages based on the values of the central source mass $M_{\star}$, the disk mass $M_{\text {disk }}$, the envelope mass $M_{\text {env }}$, and the envelope accretion rate $\dot{M}_{\text {env }}$. Stage I YSOs are those that have $\dot{M}_{\text {env }} / M_{\star}>10^{-6} \mathrm{yr}^{-1}$, i.e., protostars with large accretion envelopes; stage II are those with $\dot{M}_{\text {disk }} / M_{\star}>10^{-6} \mathrm{yr}^{-1}$ and $\dot{M}_{\text {env }} / M_{\star}<10^{-6} \mathrm{yr}^{-1}$, i.e., young objects with prominent

\footnotetext{
${ }^{1}$ http://caravan.astro.wisc.edu/protostars/
}

Table 2. Results of the SED fitting for class I YSO candidates.

\begin{tabular}{lccc}
\hline \hline Source & $\chi_{\min }^{2} / N$ & $\chi_{\text {star }}^{2} / N$ & Stage \\
\hline 1 & 0.4 & 15 & I or (II) \\
2 & 4 & 77 & II \\
3 & 0.1 & 93 & I \\
4 & 0.02 & 37 & I or (II) \\
5 & 0.9 & 4 & II or (I) \\
6 & 2.3 & 2 & II \\
7 & 10 & 91 & II \\
\hline
\end{tabular}

Notes. In the last column, we report the evolutionary stage of the source, based on the criterion of Robitaille et al. (2006) for the selected models. When the models correspond to different stages, the less likely is presented in brackets.

disks; and stage III are those with $\dot{M}_{\text {disk }} / M_{\star}<10^{-6} \mathrm{yr}^{-1}$ and $\dot{M}_{\text {env }} / M_{\star}<10^{-6} \mathrm{yr}^{-1}$, i.e., evolved sources where the flux is dominated by the central source. The goodness of the fitting for each model was measured by a $\chi^{2}$ value. We defined the "selected models" as those satisfying the equation $\chi^{2}-\chi_{\min }^{2}<2 N$, where $\chi_{\min }^{2}$ corresponds to the best-fitting model and $N$ is the number of input data fluxes (fluxes specified as upper limit do not contribute to $N$ ). The fitting tool also fit the IR fluxes to a stellar photosphere to check whether the source could be modeled by a main-sequence star with interstellar reddening. The goodness of the fitting is defined by a $\chi_{\text {star }}^{2}$ value.

In Table 2, we report the results of the SED fitting for the seven class I candidates within the molecular shell. For all of them, all the selected models correspond to stage I and II (except source 2 for which we get one stage III model). Moreover, for all of them apart from source 6 we get $\chi_{\text {min }}^{2}<\chi_{\text {star. }}^{2}$. Thus, we can confirm that they are young sources at the earlier stages of evolution.

As we note in the previous section, the molecular shell might have formed as a consequence of the interaction between the stellar wind of the central massive star and the ISM. The presence of YSO candidates within the molecular shell and an IRDC are signs of active star-forming activity. We suggest that the birth of these new stars might have been triggered by the expanding wind bubble acting on the molecular gas.

\subsection{G24.73+0.69 and its surrounding: the big picture}

Massive stars form in giant molecular clouds which, under certain circumstances, fragment into dense clumps that collapse to form new stars. These stars spend a short period of time $\left(\sim 10^{6} \mathrm{yrs}\right)$ in the MS before evolving to become LBV and WR stars, which eventually end their lifetimes exploding as supernovae. These explosions usually occur when the progenitor 


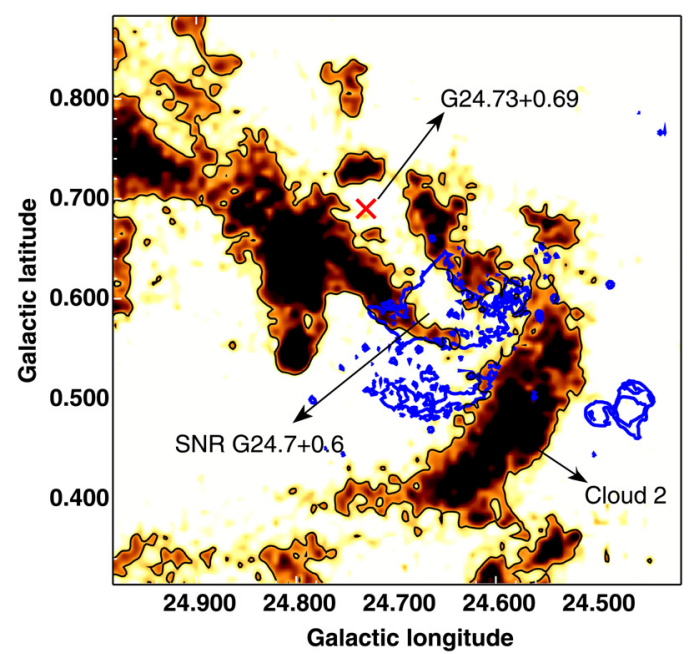

Fig. 4. Emission of the ${ }^{13} \mathrm{CO}$ integrated between +38 and $+50 \mathrm{~km} \mathrm{~s}^{-1}$. The blue contours are the radiocontinuum emission at $20 \mathrm{~cm}$ of the SNR G24.7+0.6 taken from the MAGPIS. The red cross indicates the position of the LBV star $\mathrm{G} 24.73+0.69$.

star still lies close to its natal cloud and while other companion stars may still be in the MS or LBV/WR phase. As a consequence, several supernova remnants have been observed interacting with evolved massive stars (see Velázquez et al. 2003; Sushch et al. 2011) and molecular gas (see Jiang et al. 2010, for an exhaustive list of SNR/MC associations).

The LBV star G24.73+0.69 lies $\sim 7^{\prime}$ from the SNR $\mathrm{G} 24.7+0.6$. In the radio band, this remnant displays a couple of incomplete shells and a polarized filled central core with a flat spectrum (Reich et al. 1984), which indicates that it consists of a plerion powered by an undetected pulsar. Thus, the SNR formed from the collapse of a massive star. Petriella et al. (2008) reported the existence of molecular gas interacting with G24.7+0.6 in the velocity range between +38 and $+50 \mathrm{~km} \mathrm{~s}^{-1}$, which interestingly places the SNR at the same kinematic distance as G24. In addition, active star formation probably triggered by the SNR and/or its progenitor was also discovered around the remnant (Petriella et al. 2010). In Fig. 4, we show the emission of the ${ }^{13} \mathrm{CO} J=1-0$ integrated in the velocity range between +38 and $+50 \mathrm{~km} \mathrm{~s}^{-1}$. We indicate the position of the different features in the field: the LBV star (red cross), the SNR (in blue contours), and the MC interacting with the remnant (cloud 2 in the nomenclature of Petriella et al. 2008).

From the previous figure, we see that this large region, which harbors a rich variety of objects (a LBV star and its nebula, a SNR, abundant molecular material hosting dark clouds, possible star-forming sites), is an ideal laboratory to investigate the genetic connection between different stellar populations. On the basis of the morphology and distribution of the molecular gas, it is likely that the progenitor star of the SNR G24.7+0.6 and the LBV star may have formed from the same giant MC that now we observe fragmented into smaller features. They may be the most evolved members of a so far undetected cluster/association of massive stars that also formed from the same molecular material. In addition, they may have triggered the formation of a second generation of stars that now we observe as young objects deeply embedded in the molecular gas. This scenario should be investigated further by searching for the missing intermediate-age stellar population, namely massive stars still in the MS phase, which formed together with the LBV and the supernova progenitor.

\section{Summary}

We have analyzed the ${ }^{13} \mathrm{CO}$ emission in the surroundings of the LBV star G24.73+0.69. We have discovered a fragmented molecular shell in the velocity range between $+39 \mathrm{~km} \mathrm{~s}^{-1}$ and $+44 \mathrm{~km} \mathrm{~s}^{-1}$, which delineates the infrared bipolar outer shell of G24. On the basis of spatial, morphological, and kinematic coincidence of features, we suggest that there is a connection between this IR shell and the molecular gas. We argue that the molecular shell formed from the interstellar material swept-up by the stellar wind of the central star mainly during its MS phase. The elongated morphology of the bipolar and molecular shells may be a consequence of the asymmetric stellar wind from the central massive star. We have also detected molecular emission probably associated with the inner infrared nebula, but observations of higher angular resolution are needed to spatially resolve this emission and establish its origin.

We have studied the star formation activity around G24 and discovered seven YSOs with spectral characteristics of protostars, projected on the molecular cloud that interestingly coincides with the IRDC $024.789+0.633$. Thus, this region harbors the typical components commonly found in the vicinity of star forming regions, namely an IRDC, several solar masses of molecular gas, and embedded YSO candidates. We propose that the birth of these young objects might have been triggered by the expanding stellar wind bubble.

From the study of the distribution of the molecular gas in a large region toward G24, we suggest a link between the origin of the LBV star and the progenitor star of the nearby SNR G24.7+0.6. This SNR is also interacting with the neighboring molecular gas and shows star-forming activity in its surroundings. We propose a scenario where both massive stars belong to a first generation of stars that formed from the same natal MC, pointing to the possibility of finding other massive stars with a common origin. The strong stellar winds of these massive stars might have triggered the formation of a second generation of stars, which now appear as protostars deeply embedded in the molecular gas.

Acknowledgements. We wish to thank the anonymous referee whose comments and suggestions have helped to improve the paper. A.P. is a doctoral fellow of CONICET, Argentina. S.P. and E.G. are members of the Carrera del investigador científico of CONICET, Argentina. This research was partially supported by Argentina Grants awarded by CONICET, ANPCYT and University of Buenos Aires (UBACYT)

\section{References}

Allen, L. E., Calvet, N., D’Alessio, P., et al. 2004, ApJS, 154, 363

Cappa, C., Niemela, V. S., Martín, M. C., \& McClure-Griffiths, N. M. 2005, A\&A, 436, 155

Cappa, C. E., Rubio, M., Martín, M. C., \& Romero, G. A. 2009, A\&A, 508, 759 Cappa, C. E., Vasquez, J., Pineault, S., \& Cichowolski, S. 2010, MNRAS, 403, 387

Castro-Carrizo, A., Quintana-Lacaci, G., Bujarrabal, V., Neri, R., \& Alcolea, J. 2007, A\&A, 465, 457

Cichowolski, S., Pineault, S., Arnal, E. M., \& Cappa, C. E. 2008, A\&A, 478, 443

Clark, J. S., Egan, M. P., Crowther, P. A., et al. 2003, A\&A, 412, 185

Clark, J. S., Larionov, V. M., \& Arkharov, A. 2005, A\&A, 435, 239

Clark, J. S., Crowther, P. A., Larionov, V. M., et al. 2009, A\&A, 507, 1555

de Jager, C. 1998, A\&AR, 8, 145

Fich, M., Blitz, L., \& Stark, A. A. 1989, ApJ, 342, 272

Giacani, E., Smith, M. J. S., Dubner, G., \& Loiseau, N. 2011, A\&A, 531, A138 Humphreys, R. M., \& Davidson, K. 1994, PASP, 106, 1025

Humphreys, R. M., Lamers, H. J. G. L. M., Hoekzema, N., \& Cassatella, A. 1989, A\&A, 218, L17

Jackson, J. M., Rathborne, J. M., Shah, R. Y., et al. 2006, ApJS, 163, 145

Jiang, B., Chen, Y., Wang, J., et al. 2010, ApJ, 712, 1147 
Jiménez-Esteban, F. M., Rizzo, J. R., \& Palau, A. 2010, ApJ, 713, 429

Maeder, A. 1989, in IAU Colloq., 113, Physics of Luminous Blue Variables, ed. K. Davidson, A. F. J. Moffat, \& H. J. G. L. M. Lamers, Astrophys. Space Sci. Lib., 157,15

Meaburn, J., Boumis, P., Redman, M. P., López, J. A., \& Mavromatakis, F. 2004, A\&A, 422, 603

Mellema, G., \& Lundqvist, P. 2002, A\&A, 394, 901

Meynet, G., \& Maeder, A. 2000, A\&A, 361, 101

Mokiem, M. R., de Koter, A., Vink, J. S., et al. 2007, A\&A, 473, 603

Nota, A., Livio, M., Clampin, M., \& Schulte-Ladbeck, R. 1995, ApJ, 448, 788

Nota, A., Pasquali, A., Marston, A. P., et al. 2002, AJ, 124, 2920

Petriella, A., Paron, S., \& Giacani, E. 2008, Boletin de la Asociacion Argentina de Astronomia, 51, 209

Petriella, A., Paron, S., \& Giacani, E. 2010, Boletin de la Asociacion Argentina de Astronomia, 53, 221

Prinja, R. K., Barlow, M. J., \& Howarth, I. D. 1990, ApJ, 361, 607

Rathborne, J. M., Jackson, J. M., \& Simon, R. 2006, ApJ, 641, 389
Rathborne, J. M., Simon, R., \& Jackson, J. M. 2007, ApJ, 662, 1082

Reich, W., Furst, E., \& Sofue, Y. 1984, A\&A, 133, L4

Rizzo, J. R., Jiménez-Esteban, F. M., \& Ortiz, E. 2008, ApJ, 681, 355

Robitaille, T. P., Whitney, B. A., Indebetouw, R., Wood, K., \& Denzmore, P. 2006, ApJS, 167, 256

Robitaille, T. P., Whitney, B. A., Indebetouw, R., \& Wood, K. 2007, ApJS, 169, 328

Simon, R., Jackson, J. M., Clemens, D. P., Bania, T. M., \& Heyer, M. H. 2001, ApJ, 551, 747

Smith, N., \& Owocki, S. P. 2006, ApJ, 645, L45

Sushch, I., Hnatyk, B., \& Neronov, A. 2011, A\&A, 525, A154

Velázquez, P. F., Koenigsberger, G., \& Raga, A. C. 2003, ApJ, 584, 284

Weis, K. 2001, in Eta Carinae and Other Mysterious Stars: The Hidden Opportunities of Emission Spectroscopy, ed. T. R. Gull, S. Johannson, \& K. Davidson, ASP Conf. Ser., 242, 129

Whitworth, A. P., Bhattal, A. S., Chapman, S. J., Disney, M. J., \& Turner, J. A. 1994, MNRAS, 268, 291 